\title{
Susceptibilidad genética y riesgo de cáncer gástrico en una población del Cauca
}

\begin{abstract}
María M. Torres, Claudia P. Acosta, Diana M. Sicard, Helena Groot de Restrepo
Laboratorio de Genética Humana, Facultad de Ciencias, Universidad de los Andes, Bogotá, D.C., Colombia.

El cáncer gástrico es la principal causa de mortalidad por cáncer en Colombia. El riesgo de desarrollar cáncer gástrico se ha asociado con factores ambientales y con la infección por Helicobacter pylori. Las enzimas glutatión-S-transferasas están involucradas en la desintoxicación de varios carcinógenos ambientales. Las deleciones homocigóticas de glutatión-S-transferasa M1 (GSTM1-0) y glutatión-S-transferasa T1 (GSTT1-0) se han asociado con algunos tipos de cáncer. Los niveles del factor de necrosis tumoral (FNT $\alpha$ ) están aumentados en pacientes infectados por H. pylori. Una transición G/A en la posición -308 del promotor del FNT- $\alpha$ se ha visto relacionada en algunos estudios con un incremento en la expresión del gen, y está asociada con la susceptibilidad a cáncer gástrico. Se investigó la asociación de estos polimorfismos con cáncer gástrico y la interacción con otros factores de riesgo (estilo de vida). Se obtuvieron muestras de sangre de 46 pacientes con cáncer gástrico y 96 controles. Se empleó el modelo de regresión logística para obtener la razón de posibilidades (OR) y sus intervalos de confianza del 95\% y, así, establecer la asociación entre los polimorfismos enzimáticos y el cáncer gástrico, y entre otros factores independientes y esta enfermedad. Las frecuencias de los polimorfismos de deleción en pacientes y controles fueron: para la GSTM1, $65,2 \%$ y $37,5 \%$, y para la GSTT1, $17,4 \%$ y $14,6 \%$, respectivamente. La frecuencia del polimorfismo G/A del FNT $\alpha$ en las personas infectadas con $H$. pylori fue de $18 \%$ en la población con cáncer gástrico y de $7 \%$ en el grupo control. Nuestros resultados sugieren que el polimorfismo de deleción de GSTM1 puede estar asociado con un riesgo aumentado de cáncer gástrico (OR $5,5 ; \mathrm{IC} 95 \%, 1,7-17,2)$. Igualmente, nuestros datos muestran que otros factores de riesgo como la infección por $\mathrm{H}$. pylori y el consumo de cigarrillo y alcohol están asociados con este tipo de cáncer (OR 5,58; IC95\% 1,81-17,19; OR 6,70; IC95\%, 2,20-20,30 y OR 3,27; IC95\% 1,14-9,4, respectivamente).
\end{abstract}

Palabras clave: cáncer gástrico, glutatión-S-transferasas, polimorfismos, Colombia.

Genetic susceptibility and risk of gastric cancer in a human population of Cauca, Colombia

Gastric cancer (GC) is the main cause of mortality by cancer in Colombia. Glutathione Stransferase (GST) enzymes are involved in the detoxification of many environmental carcinogens. The homozygous deletions of glutathione S-transferase M1 (GSTM1-0) and glutathione Stransferase T1 (GSTT1-0) have been associated with several types of cancer. The risk to develop GC has been associated with environmental factors and Helicobacter pylori infection. The tumor necrosis factor (TNF- $\alpha$ ) and its levels are increased in patients infected with H. pylori. A G/ A transition in the position -308 of the promoter of the TNF- $\alpha$ has been related in several studies to an increased expression of the gene and is associated with susceptibility to GC. The association of these polymorphisms with $\mathrm{GC}$ and the interaction with other risk factors (life style) were investigated. Blood samples were obtained from 46 GC patients and 96 controls. The logistic regression model was used to obtain the odds ratio (OR) and their $95 \%$ confidence intervals. These statistics established the association between the enzymatic polymorphisms and GC and between other independent factors and GC. The frequency of the TNF- $\alpha$ polymorphism in people infected with $H$. pylori was $18 \%$ in the GC population and $7 \%$ in the control group. This transition was not significantly associated with $H$. pylori infection and GC. The frequencies of the deletion polymorphisms for patients and controls were as follows: GSTM1 $65.2 \%$ and $37.5 \%$; GSTT1 $17.4 \%$ and $14.6 \%$. These results suggested that the GSTM 1 deletion 
polymorphism was associated with an increased risk of gastric cancer (OR of $5.5 ; 95 \% \mathrm{Cl}, 1.7$ 17.2). Furthermore, other risk factors such as $H$. pylori infection (OR 5.58,Cl 1.8-17.2), smoking (OR 6.70, Cl 2.2-20.3) and alcohol intake (OR 3.27, Cl 1.1-9.4) were associated with GC.

Key words: gastric cancer, glutathione S-transferase, polymorphisms, Colombia.

El cáncer gástrico es una de las principales causas de mortalidad por cáncer en el mundo. Si bien su incidencia ha disminuido en los últimos 50 años (1), sigue siendo el cáncer más frecuente de los países en vías de desarrollo, aunque posee tasas de incidencia variables entre los diferentes países. El tipo de cáncer más frecuente es el adenocarcinoma $(90 \%)$, con una variante intestinal predominantemente distal y otra difusa.

Entre los factores reconocidos de riesgo en el cáncer gástrico se destacan: la gastritis crónica atrófica con metaplasia intestinal, la infección por Helicobacter pylori, las dietas ricas en alimentos salados, ahumados o secos, el tabaquismo, la anemia perniciosa, la enfermedad de Menetrier, la poliposis familiar, el sexo masculino y la edad avanzada.

En Latinoamérica, las tasas de incidencia reportadas según el sexo son variables (2); las mayores son en Costa Rica (51,5 por 100.000 en hombres y 28,7 por 100.000 en mujeres) y las menores en Perú (19,1 por 100.000 en hombres y 13,7 por 100.000 en mujeres). En Colombia, según el Instituto Nacional de Cancerología, el cáncer gástrico es la primera causa de muerte por cáncer en los hombres y la tercera en las mujeres, precedida por los cánceres de cuello uterino y de mama. La distribución geográfica de las tasas de mortalidad es variable; son altas en los departamentos del centro y oriente del país (Santander, Norte de Santander, Cundinamarca, Boyacá, Nariño, etc.) y bajas en los departamentos de la Costa Atlántica y los Llanos Orientales. La edad promedio de mortalidad es de 59 años y la relación de mortalidad por sexo (hombre/mujer) es de 1,7 (3).

\footnotetext{
Correspondencia:

María M. Torres, Laboratorio de Genética Humana, Universidad de los Andes, apartado aéreo 4976, Bogotá, D.C., Colombia

Teléfono: 339 4949, extensión 2790; fax: 3394949 ext. 2817

Correo electrónico: maritorr@uniandes.edu.co

Recibido: 01/09/03; aceptado:27/05/04
}

En Colombia se han realizado algunos estudios epidemiológicos en cuanto a los factores de riesgo para esta patología (4). Numerosos autores han indicado entre los principales riesgos, además de la presencia de $H$. pylori, la conservación de alimentos con sal o ahumados, asociada con una alimentación pobre en frutas y verduras, y a un nivel socioeconómico bajo (5).

La variación individual en riesgo de cáncer ha sido asociada con variantes alélicas específicas de diferentes genes (polimorfismos) que se encuentran presentes en una amplia proporción de la población normal. Estos polimorfismos en una gran variedad de genes pueden modificar el efecto de la exposición ambiental (6) y esta interacción gen-ambiente podría explicar la alta variación en la incidencia de cáncer gástrico en el mundo (7).

La susceptibilidad genética puede ser crítica en muchos de los pasos de la carcinogénesis gástrica. Esta susceptibilidad se debe a la presencia de variantes alélicas de genes involucrados en: 1) factores de protección de la mucosa gástrica (mucinas); 2) factores inmunogenéticos como la respuesta inflamatoria inducida por citocinas (IL-1 $\beta$ y FNT $\alpha$ ); 3) variabilidad intrínseca en las proteínas involucradas en los mecanismos de reparación de ADN (XRCC1), y 4) variabilidad en la respuesta a la desintoxicación de compuestos carcinógenos llevada a cabo por las enzimas metabólicas (8).

La interacción entre los factores ambientales y la susceptibilidad genética se ha analizado principalmente con genes involucrados en el metabolismo de compuestos xenobióticos y carcinógenos (9). Existe un gran número de enzimas metabólicas que pueden ser agrupadas dentro de familias como: citocromo p450 (fase I) que usualmente lideran la activación de compuestos carcinógenos y glutatión Stransferasas y $\mathrm{N}$-acetiltransferasas (fase II), que actúan en la desintoxicación o activación de 
compuestos carcinógenos. De estas enzimas de fase II, los genes más estudiados son el GSTM1 y GSTT1. La ausencia de alelos que codifican estas enzimas provenientes de la madre y del padre (polimorfismo de deleción, GSTM1-0 o GSTT1-0) se ha asociado con un alto riesgo de cáncer gástrico debido a la poca capacidad de desintoxicar compuestos carcinógenos (10-13).

Las diferencias individuales en cuanto a la intensidad en la respuesta inflamatoria pueden contribuir a la transformación de la mucosa gástrica. El factor de necrosis tumoral (FNT) es una importante y potente citocina proinflamatoria. Los niveles de FNT se han visto incrementados en pacientes infectados con $\mathrm{H}$. pylori, probablemente porque se encuentra relacionado con la secreción de ureasa $(14,15)$. Se han descrito variantes alélicas para este gen y una de las más reconocidas es la transición de $\mathrm{G}$ por A encontrada en la posición -308 del promotor. La expresión aumentada de este gen se ha visto involucrada en una alta susceptibilidad ante el desarrollo de enfermedades autoinmunes, infecciosas y de cáncer gástrico (16). Sin embargo, no en todos los estudios se ha visto asociación entre la producción de FNT $\alpha$ dependiendo del genotipo 308 (17).

En países como Colombia, en donde existe una gran incidencia de cáncer gástrico, han sido pocos los estudios que han buscado correlacionar algunos factores ambientales y de susceptibilidad genética con el riesgo de adquirir este cáncer (1822). El objetivo del presente estudio fue establecer la asociación entre las variantes genéticas de enzimas metabólicas (GSTM1, GSTT1) y del FNT $\alpha(-308 \mathrm{~A})$ y el riesgo de cáncer gástrico. De igual forma, se evaluó la interacción entre algunos factores de riesgo como la infección con H. pylori, el consumo de alcohol, el cigarrillo, algunos hábitos alimentarios y el cáncer gástrico.

\section{Materiales y métodos}

\section{Selección de los controles y pacientes con cáncer gástrico}

Se analizaron 50 pacientes de la Unidad de Gastroenterología del Hospital Susana López de Valencia de Popayán con diagnóstico patológico confirmado de adenocarcinoma gástrico en el 2000.
Los criterios de inclusión para los pacientes fueron: 1) sin restricción por edad; 2) condición médica y física estable; 3) libre aceptación para hacer parte del estudio (consentimiento informado). Los controles fueron 96 individuos que asistieron a la Unidad de Gastroenterología con diagnóstico diferente a cáncer gástrico y se seleccionaron de acuerdo con el rango de edad y el sexo de los pacientes con cáncer gástrico.

\section{Diagnóstico histológico}

Los pacientes y los controles fueron sometidos a una endoscopia gastrointestinal diagnóstica, en la cual se examinaron y tomaron biopsias de los sitios definidos de importancia clínica. La infección por $H$. pylori fue diagnosticada en el estudio histopatológico.

\section{Recolección de los datos}

Se diligenció un cuestionario estandarizado a los pacientes y a los controles. La información recolectada incluyó: 1) factores demográficos; 2) historia ocupacional; 3) historia familiar de cáncer; 4) consumo de sal y carnes ahumadas en los últimos cinco años; 5) consumo de alcohol y cigarrillo; 6) otros hábitos alimentarios. Se adicionaron datos provenientes de las historias clínicas que incluían la información de la endoscopia y la patología. Se obtuvieron muestras de sangre total de 46 pacientes con cáncer gástrico y 96 controles, a las cuales se les realizaron las pruebas de genotipificación de las enzimas metabólicas GSTM1 y GSTT1 y del FNT.

\section{Extracción de ADN y análisis de las variantes genéticas de las enzimas metabólicas (GSTM1 y GSTT1)}

El ADN genómico fue extraído a partir de $8 \mu \mathrm{l}$ de sangre total utilizando la resina de intercambio iónico Chelex 100 (Biorad, Hercules, CA, USA) según el protocolo de Walsh et al. (23). Las variantes genéticas de GSTM1 y GSTT1 se determinaron simultáneamente en una misma reacción de amplificación, según la metodología propuesta por Abdel-Rahman et al.(24). Cincuenta ng del ADN extraído fueron amplificados en $25 \mu \mathrm{l}$ de una mezcla de reacción que contenía 30 pmol de cada uno de los siguientes iniciadores para 
GSTM1: 5'GAA CTC CCT GAA AAG CTA AAG 3' y 5'GTT GGG CTC AAA TAT ACG GTG G 3' ; y para GSTT1: 5'TTC CTT ACT GGT CCT CAC ATC TC 3' y 5'TCA CCG GAT CAT GGC CAG CA 3'. Como control interno se amplificó el gen CYP1A1 con los siguientes iniciadores: 5'GAA CTG CCA CTT CAG CTG TCT 3' y 5'CAG CTG CAT TTG GAA GTG CTC 3'. El ADN y los iniciadores fueron amplificados en presencia de $200 \mu \mathrm{mol}$ dNTP, $5 \mu \mathrm{l}$ de solución tamponada 10x $(10 \times 500 \mathrm{mM} \mathrm{KCl}$, $100 \mathrm{mM}$ Tris- $\mathrm{HCl}, \mathrm{pH} 9,0$ ) y $1,5 \mathrm{mM} \mathrm{MgCl}{ }_{2}$ y $2 \mathrm{U}$ Amplitaq ADN polimerasa (Promega, Madison, $\mathrm{WI}$, USA). Las condiciones de amplificación fueron las siguientes: $94^{\circ} \mathrm{C}$ por cinco minutos, seguidos de 35 ciclos de $94^{\circ} \mathrm{C}$ por dos minutos, $59^{\circ} \mathrm{C}$ por un minuto y $72^{\circ} \mathrm{C}$ por un minuto, y un paso final de extensión de $72^{\circ} \mathrm{C}$ por diez minutos. Los productos amplificados se visualizaron con bromuro de etidio en gel de agarosa (FMC BioProducts, Rockland, ME, USA) al $2 \%$. La presencia o ausencia de una banda de $480 \mathrm{pb}$ corresponde al gen GSTT1 y una banda de $215 \mathrm{pb}$ al gen GSTM1; de igual forma, una banda de $312 \mathrm{pb}$ corresponde al gen CYP1A1.

\section{Factor de necrosis tumoral alfa (-308 A $F N T \alpha$ )}

La amplificación enzimática para la identificación del polimorfismo G-308A del FNT $\alpha$ se llevó a cabo según el protocolo descrito por DiGiovine (25). Los iniciadores utilizados fueron los siguientes: 5 ' AGG CAA TAG GTT TTG AGG GCC AT 3' y 5' TCC TCC CTG CTC CGA TTC CG 3'. El producto de amplificación genera un fragmento de $107 \mathrm{pb}$, el cual es digerido por una enzima de corte $\mathrm{Ncol}$ (Promega, Madison, WI, USA), la cual permite detectar la transición de $\mathrm{G}$ por $\mathrm{A}$. La digestión enzimática se produce en presencia del alelo normal $(G)$ y genera dos bandas, una de $87 \mathrm{pb}$ y otra de $20 \mathrm{pb}$. El alelo raro (A) no es cortado lo cual genera una banda de $107 \mathrm{pb}$. Los individuos homocigotos normales presentan una sola banda de $87 \mathrm{pb}$, los heterocigotos $A / G$ dos bandas, 87 y $107 \mathrm{pb}$, y los homocigotos para el alelo raro (A) una banda de $107 \mathrm{pb}$.

\section{Análisis estadísticos}

La asociación entre el cáncer gástrico y los polimorfismos de deleción de las enzimas GSTM1 y GSTT1, así como el polimorfismo del FNT, se calcularon mediante la razón de posibilidades (odds ratio, OR) y los intervalos de confianza del $95 \%$, utilizando el paquete estadístico Stata 7.0. Igualmente, se investigó la asociación de otros factores de riesgo y cáncer gástrico. Los OR y su IC95\% se obtuvieron usando un modelo de regresión logística.

\section{Resultados}

El cuadro 1 muestra las características generales de la población de estudio al igual que la distribución de variables como edad, sexo, consumo de alcohol, cigarrillo y carnes ahumadas, nivel de escolaridad, procedencia e infección con

Cuadro 1. Características generales de la población en estudio.

\begin{tabular}{|c|c|c|c|c|}
\hline & & Casos & Cor & oles \\
\hline & $\mathbf{n}$ & & $\mathbf{n}$ & $(\%)$ \\
\hline Grupos de edad (a) & & & & \\
\hline$\leq 40$ & 3 & (6) & 5 & $(5,2)$ \\
\hline $41-50$ & 10 & (20) & 24 & $(25,0)$ \\
\hline $51-60$ & 13 & (26) & 23 & $(24,0)$ \\
\hline$\geq 60$ & 24 & (48) & 44 & $(45,8)$ \\
\hline Total & 50 & & 96 & $(100)$ \\
\hline Sexo & & & & \\
\hline Masculino & 25 & $(50)$ & 41 & $(42,7)$ \\
\hline Femenino & 25 & (50) & 55 & $(57,3)$ \\
\hline Total & 50 & $(100)$ & 96 & $(100)$ \\
\hline Consumo de alcoh & & & & \\
\hline No & 25 & (50) & 67 & $(69,8)$ \\
\hline Sí & 25 & (50) & 29 & $(30,2)$ \\
\hline Fumar & & & & \\
\hline No & 21 & (42) & 58 & $(60,4)$ \\
\hline Sí & 29 & (58) & 38 & $(39,6)$ \\
\hline Comidas ahumadas & & & & \\
\hline No & 11 & (22) & 42 & $(44,0)$ \\
\hline Sí & 39 & (78) & 54 & $(56,0)$ \\
\hline Infección con $H$. py & & & & \\
\hline No & 35 & (70) & 65 & $(67,7)$ \\
\hline Sí & 15 & (30) & 31 & $(32,3)$ \\
\hline Origen & & & & \\
\hline Urbano & 18 & (36) & 84 & $(87,5)$ \\
\hline Rural & 32 & (64) & 12 & $(12,5)$ \\
\hline Educación & & & & \\
\hline Sin educación & 9 & (18) & 0 & \\
\hline Educación primaria & 27 & (54) & 26 & $(27,1)$ \\
\hline Media & 9 & (18) & 45 & $(46,9)$ \\
\hline Superior & 5 & (10) & 25 & $(26,0)$ \\
\hline
\end{tabular}


H. pylori. El promedio de edad de los pacientes con cáncer gástrico y de los controles fue de 60 años en los hombres y de 58 años para las mujeres. Particularmente en este estudio, se observó un número similar de hombres y de mujeres con cáncer gástrico, diferente a lo reportado en la literatura en el cual es mayor la frecuencia en el sexo masculino. Muchos de los pacientes provenían de áreas rurales cercanas a Popayán y con un nivel de escolaridad primaria.

La frecuencia de GSTM1-0 y de GSTT1-0, al igual que su asociación con cáncer gástrico se muestran en el cuadro 2. La prevalencia del polimorfismo de deleción de GSTM1 fue muy alta en los pacientes con cáncer gástrico $(65,2 \%)$ comparado con el grupo control $(37,5 \%)$. No se observó ninguna diferencia entre la prevalencia del polimorfismo de deleción de GSTT1 entre los casos $(17,4 \%)$ y los controles $(14,6 \%)$.

El cuadro 2 muestra los resultados de la razón de posibilidades y de los IC95\% de GSTM1-0 y GSTT1-0 comparando los pacientes con cáncer gástrico y el grupo control. Teniendo en cuenta el genotipo normal para cada una de las enzimas como el de referencia, se encontró una asociación significativa entre GSTM1-0 y cáncer gástrico, $\mathrm{OR}=3,12(\mathrm{IC} 95 \%, 1,41-7,0), p=0,002)$. No se halló ninguna asociación con GSTT1-0, OR=1,23 (IC95\%, 0,41-3,47). Después de controlar las variables de confusión como edad, sexo, nivel de escolaridad, antecedentes familiares de cáncer, consumo de carnes ahumadas, consumo de alcohol y cigarrillo al igual que la infección con $\mathrm{H}$. pylori, el OR ajustado para los genotipos GSTM10 y GSTT1-0 fue de 5,45 (IC95\%, 1,72-17,20) y 0,47 (IC95\%, 0,09-2,27), respectivamente.

El cuadro 3 muestra los resultados de la razón de posibilidades y los intervalos de confianza del $95 \%$ de los polimorfismos del FNT $\alpha$ - 308 comparando los pacientes con cáncer y el grupo control. EI análisis de la distribución alélica del FNT $\alpha-308$ en el grupo control mostró que se encuentra en equilibrio de Hardy-Weinberg. Teniendo en cuenta el genotipo G/G como el alelo común, no se encontró una asociación entre los polimorfismos de FNT $\alpha$ y cáncer gástrico. El cuadro 4 muestra

Cuadro 2. Relación entre los genotipos GSTM1 y GSTT1 y cáncer gástrico.

\begin{tabular}{|c|c|c|c|c|}
\hline & Controles $(n=96)$ & Cáncer gástrico $(n=46)$ & OR (IC 95\%) ${ }^{a}$ & OR (IC 95\%) ${ }^{b}$ \\
\hline \multicolumn{5}{|l|}{ GSTM1 } \\
\hline Presente & $60(62,5)$ & $16(34,1)$ & & \\
\hline Deleción & $36(37,5)$ & $30(65,2)$ & $3,12(1,41-7,00)$ & $5.45(1,72-17,20)$ \\
\hline \multicolumn{5}{|l|}{ GSTT1 } \\
\hline Presente & $82(85,4)$ & $38(82,6)$ & & \\
\hline Deleción & $14(14,6)$ & $8(17,4)$ & $1,23(0,54-3,23)$ & $0,47(0,09-2,27)$ \\
\hline
\end{tabular}

a Razón de posibilidades (odds ratio, OR) cruda e intervalo de confianza de 95\% (IC 95\%)

b OR e IC95\% ajustado por edad, sexo, educación, familia con cáncer, ingestión de comida ahumada, consumo de cigarrillo, consumo de alcohol e infección con H. pylori.

Cuadro 3. Relación entre los genotipos del FNT $\alpha$ G-308 y cáncer gástrico.

\begin{tabular}{lccll}
\hline & Controles $n=66(\%)$ & CG $n=44(\%)$ & OR (IC95\%) & OR (IC95\%) \\
\hline Genotipo & & & & \\
G/G & $56(85,0)$ & $41(93,0)$ & 1,0 & 1,0 \\
A/G & $10(15,0)$ & $3(7,0)$ & $0,41(0,10-1,58)$ & $0,32(0,05-2,08)$ \\
A/A & $0(0)$ & $0(0)$ & & \\
\hline
\end{tabular}

Los polimorfismos del FNT $\alpha$ corresponden a los genotipos: G/G (común), A/G (heterocigoto) y A/A (raro). El análisis de frecuencias alélicas en población control muestra equilibrio de Hardy-Weinberg $\left(c^{2}=0,44, p>0,05\right)$.

a Razón de posibilidades (odds ratio, OR) cruda e intervalo de confianza del 95\% (IC95\%)

b OR e IC95\% ajustado por edad, sexo, educación, familia con cáncer, ingestión de comida ahumada, consumo de cigarrillo, consumo de alcohol e infección con H. pylori. 
Cuadro 4. Relación entre los factores de riesgo y cáncer gástrico.

\begin{tabular}{lll}
\hline Variables & OR (IC95\%) & OR $\mathbf{~ ( I C 9 5 \% ) ~}^{\mathbf{b}}$ \\
\hline Edad & $0,96(0,93-1,00)$ & $0,96(0,92-1,00)$ \\
Antecedentes de cáncer & $0,47(0,10-2,15)$ & - \\
Alimentos ahumados & $3,05(0,87-10,64)$ & $2,04(0,76-7,96)$ \\
Hábitos de fumar & $5,90(2,00-17,00)^{*}$ & $6,70(2,20-20,30)^{\star}$ \\
Consumo de alcohol & $2,30(0,70-7,50)$ & $3,27(1,14-9,40)^{*}$ \\
Infección con H. pylori & $5,57(1,74-17,75)^{\star}$ & $5,58(1,81-17,19)^{\star}$ \\
Origen (rural o urbano) & $0,09(0,02-0,37)$ & $0,12(0,03-0,40)$ \\
Sin educación & $3,43(0,75-15,68)$ & $3,00(0,68-13,21)$ \\
Educación primaria & $2,40(0,48-11,85)$ & $1,91(0,41-8,75)$ \\
\hline
\end{tabular}

a Razón de posibilidades (odds ratio, OR) ajustada por edad, sexo, educación, antecedentes familiares de cáncer, comidas ahumadas, consumo de alcohol y cigarrillo y la infección con $H$. pylori.

b Razón de posibilidades (odds ratio, OR) ajustada por edad, educación, comidas ahumadas, consumo de alcohol, consumo de cigarrillo y la infección con $H$. pylori.

${ }^{*} p<0,01$

los resultados de la razón de posibilidades y los intervalos de confianza del $95 \%$ de algunos factores de riesgo asociados con cáncer gástrico, comparando los pacientes y el grupo control. Se encontró una asociación grande entre los pacientes infectados con $\mathrm{H}$. pylori y la presencia de cáncer gástrico, OR=5,57; IC95\%, 1,74-17,75. De igual forma, otros factores de riesgo como el consumo de alcohol y de cigarrillo muestran asociación con el cáncer gástrico, $\mathrm{OR}=3,27$; IC95\%, 1, 14-9,40 y OR=5,90; IC95\%, 2,00-17,00), respectivamente.

\section{Discusión}

Los polimorfismos genéticos de enzimas involucradas en el metabolismo de compuestos xenobióticos han sido considerados como un factor de riesgo importante en la susceptibilidad en diferentes tipos de cáncer, según lo demuestran varios estudios de casos y controles (26-28). En el presente estudio se observó una asociación importante entre el genotipo de deleción de la enzima GSTM1 y el cáncer gástrico $(65,2 \%$, en los casos y $37,5 \%$, en los controles). Esto puede ser ocasionado porque la ausencia de este gen no permite la síntesis de esta enzima, la cual está involucrada en el proceso de desintoxicación de compuestos químicos como los hidrocarburos poliaromáticos y la nitrosaminas que se encuentran en el humo del cigarrillo, los alimentos, los compuestos agroquímicos y las drogas antineoplásicas (28). Los estudios en otras poblaciones con alta incidencia de cáncer gástrico en los que se ha analizado el polimorfismo de deleción de GSTM1 y su asociación con la enfermedad, han mostrado resultados contradictorios (cuadro 5). Por otro lado, para el genotipo de deleción de GSTT1 no se encontró asociación con cáncer gástrico ( $17,4 \%$ en los casos y $14,6 \%$ en los controles). Los estudios que han investigado también la relación de GSTT1 y cáncer gástrico han arrojado igualmente resultados conflictivos $(13,22,29,33-36)$. Esto puede deberse al efecto combinado de la variación en la frecuencia de estos polimorfismos entre diferentes grupos étnicos (37) y la exposición a distintos factores ambientales sobre los cuales actúa la GST. En un estudio previo en el que se analizó la frecuencia de polimorfismos de deleción de GST en 219 personas sanas provenientes en su mayoría del altiplano cundiboyacense, se observó una frecuencia del 55\% para GSTM1-0 y del 25\% para GSTT1-0 (38). En el ámbito mundial, la frecuencia de GSTM1-0 en individuos sanos varía entre $23 \%$ y $41 \%$ en la población afroamericana y africana, $33 \%$ y $69 \%$ en asiáticos, $39 \%$ y $62 \%$ en europeos y $51 \%$ y $54 \%$ en australianos (39). Los estudios realizados en siete poblaciones amerindias brasileñas muestran rangos de frecuencia para estos genes de $4 \%$ a $43 \%$ para la GSTM1 y de $0 \%$ a $30 \%$ para la GSTT1 (40). Esto demuestra la variabilidad en la frecuencia de estos polimorfismos en diferentes poblaciones y regiones geográficas. Es de 
Cuadro 5. Estudios de casos y controles y riesgo de cáncer gástrico analizando los polimorfismos de deleción de la enzima GSTM1.

\begin{tabular}{lccccc}
\hline Población & n casos & n controles & OR & IC95\% & Referencia \\
\hline Iraníes & 42 & 131 & 2,3 & $1,15-4,95$ & 29 \\
Chinos & 146 & 429 & 0,91 & $0,57-4,45$ & 13 \\
Japoneses & 139 & 126 & 1,70 & $1,05-2,76$ & 12 \\
Japoneses & 91 & 55 & 1,68 & - & 30 \\
Japoneses & 284 & 284 & 1,17 & $0,8-1,7$ & 31 \\
Ingleses & 136 & 577 & NA & - & 32 \\
Poloneses & 304 & 427 & NA & $0,7-1,3$ & 33 \\
Colombianos (Antioquia) & 71 & 71 & - & 22 \\
\hline
\end{tabular}

NA: sin asociación

entender que el origen de la población colombiana es altamente heterogéneo, ya que está compuesta de la mezcla de poblaciones autóctonas (amerindias) y de inmigrantes de origen africano y europeo. Por lo tanto, es posible encontrar diferencias en las frecuencias de estos polimorfismos entre las diferentes poblaciones colombianas. Las diferencias en cuanto a la prevalencia de estos genotipos de deleción y de otras variantes genéticas pueden hacernos entender el porqué en algunas regiones del país el riesgo a determinado tipo de cáncer es mayor que en otras. A esto debe sumarse la variación en hábitos alimentarios que se observa en los distintos departamentos del país. El proceso de carcinogénesis gástrica ha sido descrito como un evento generado por múltiples factores. Se han mencionado los de origen genético, pero, además, en el presente estudio se encontraron asociaciones con otros factores medioambientales como son: la infección con $H$. pylori y el consumo de alcohol y cigarrillo. Cerca del $50 \%$ de la población mundial se encuentra infectada con H. pylori (41). Esta bacteria ha sido asociada con diferentes enfermedades gastrointestinales, dentro de las cuales se incluye el cáncer gástrico (42). H. pylori fue clasificado en 1994 por la Agencia Internacional de Investigación sobre el Cáncer (IARC), como carcinógeno de clase I (43). Muchos estudios demuestran que los individuos que están infectados con esta bacteria y son positivos para la cepa Cag A tienen mayor riesgo de adquirir este tipo de cáncer $(\mathrm{OR}=28,4$; IC95\%, 3,7-217,1) (44). Durante la infección con H. pylorise produce una estimulación de citocinas como IL-1, IL-6, IL-8 y FNT $\alpha$ que se encargan de aumentar la inflamación y producir daño en la mucosa gástrica (41). Algunos estudios han descrito variantes alélicas para los genes que codifican las citocinas antes mencionadas; una de las más estudiadas es la transición de G por $A$ encontrada en la posición -308 del promotor del FNT $\alpha$. Este polimorfismo en algunos casos genera una expresión aumentada del gen, lo cual hace que exista mayor daño en el tejido gástrico (16). Sin embargo, en el presente estudio no se encontró ninguna asociación entre este polimorfismo y el riesgo de cáncer gástrico. Otros factores de riesgo externos, como el ser fumador y el consumo de alcohol, han mostrado asociaciones muy altas con el riesgo de cáncer gástrico $(34,45)$. En el presente estudio se observó una asociación entre el polimorfismo de deleción de GSTM1 y el consumo de cigarrillo en los pacientes con cáncer gástrico. Estos resultados son concordantes con los encontrados en pacientes chinos con esta enfermedad (34). En el presente estudio se observó una asociación entre el consumo de alcohol y el riesgo de cáncer gástrico, lo cual ya ha sido documentado (46). Esta asociación no fue afectada por los polimorfismos de deleción, como en el caso del consumo de cigarrillo, posiblemente por no participar las enzimas GST directamente en el metabolismo del alcohol.

En conclusión, nuestros resultados sugieren que la deleción homocigota de GSTM1 en la población del Cauca puede estar asociada con una mayor susceptibilidad a cáncer gástrico; de igual forma, otros factores como la infección con H. pylori, el consumo de cigarrillo y de alcohol parecen contribuir al desarrollo de esta enfermedad. La 
asociación entre GSTM1-0 y el consumo de cigarrillo con mayor riesgo de cáncer gástrico pone de manifiesto la interacción entre los factores genéticos y los ambientales en el proceso de carcinogénesis gástrica. Una debilidad del presente estudio es el bajo número de casos, lo cual puede limitar la capacidad para estimar eficazmente el OR. No obstante, se pudo detectar la asociación entre GSTM1-0 y el cáncer gástrico. El consumo de alcohol en el presente estudio se asoció con cáncer gástrico; sería de gran interés determinar los polimorfismos de las enzimas involucradas en el metabolismo del alcohol, que pudieran conferir una mayor susceptibilidad al cáncer gástrico. Debido a la variabilidad étnica encontrada en Colombia, se resalta la necesidad de desarrollar estudios en poblaciones de diverso origen genético y con distintos estilos de vida (factor ambiental), en los que se investiguen las frecuencias de polimorfismos relevantes en el metabolismo de xenobióticos.

\section{Agradecimientos}

Este trabajo fue realizado gracias al apoyo financiero del Comité de Investigaciones de la Facultad de Ciencias de la Universidad de los Andes. Los autores quieren manifestar de manera especial sus agradecimientos a los pacientes y al grupo médico de la Unidad de Gastroenterología del Hospital Susana López de Popayán. A Carolina Arias, estudiante de la maestría en Ciencias Biológicas de la Universidad de los Andes, por las determinaciones del FNT- $\alpha$. A Nelsy Rodríguez por su colaboración en los análisis estadísticos.

\section{Referencias}

1. Coleman MP, Esteve J, Damiecki P, Arslan A, Renard $\mathrm{H}$. Trends in cancer incidence and mortality. IARC Scientific Publ. 121. Lyon, France: International Agency for Research on Cancer; 1993.

2. Parkin DM, Whelan SL, Ferlay J, Raymond L, Young J. Cancer incidence in five continents. Vol. VII. Lyon: IARC Scientific Publ. 143. Lyon, France: International Agency for Research on Cancer; 1997.

3. Instituto Nacional de Cancerología. Boletín Informativo 2001.

4. Bravo MD, Cortés A, Carrascal E, Correa P, Ordóñez N. Seroprevalencia de anticuerpos anti-Helycobacter pylori en donantes de sangre de regiones colombianas con diferencias en la mortalidad por cáncer gástrico. Colombia Médica 2000;31:122-30.
5. Nomura A. Stomach cancer. En: Schottenfeld D, Fraumeni Jr JF, editors. Cancer epidemiology and prevention. New York: Oxford University Press; 1996. p.707-24.

6. Perera FP, Weistein IB. Molecular epidemiology: recent advances and future directions. Carcinogenesis 2000; 21:517-24.

7. Ames BN. Cancer prevention and diet: help from single nucleotide polymorphisms. Proc Natl Acad Sci USA 1999;96:12216-8.

8. El-Omar EM, Chow WH, Rabkin CS. Gastric cancer and $H$. pylori: host genetics open the way. Gastroenterology 2001;121:1002-5.

9. Garte S. Metabolic susceptibility genes as cancer risk factors: time for a reassessment? Cancer Epidemiol Biomark Prev 2001;10:1233-7.

10. Strange RC, Matharoo B, Faulder GC, Jones P, Cotton W, Elder JB, et al. The human glutathione Stransferase: a case-control study of the incidence of the GST1 0 phenotype in patients with adenocarcinoma. Carcinogenesis 1991;12:25-8.

11. Harada S, Misawa S, Nakamura T, Tanaka N, Ueno E., Nozoe M. Detection of GST1 gene deletion by the polymerase chain reaction and its possible correlation with stomach cancer in Japanese. J Hum Genet 1992; 90:62-4.

12. Katoh T, Nagata N, Kuroda $\mathbf{Y}$, Itoh H, Kawara A, Kuroki N, et al. Gluthation S-transferase M1 (GSTM1) and T1 (GSTT1) genetic polymorphism and susceptibility to gastric and colorectal adenocarcinoma. Carcinogenesis 1996;17:1855-9.

13. Setiawan VW, Zhang Z-F, Yu G-P, Li Y-L, Lu M-L, Tsai C-J, et al. GSTT1 and GSTM1 null genotypes and the risk of gastric cancer: case-control study in a Chinese population. Cancer Epidemiol Biomark Prev 2000;9:73-80.

14. Noach LA, Bosma NB, Jansen J, Hoek FJ, van Deventer SJ, Tygat GN. Mucosal tumor necrosis factor-alpha, interleukin-1 beta, and interleukin-8 production in patients with Helicobacter pylori infection. Scand J Gastroenterol 1994;29:425-9.

15. Harris PR, Mobley HL, Pérez-Pérez GI, Blaser MJ, Smith PD. Helicobacter pylori urease is a potent stimulus of mononuclear phagocyte activation and inflammatory cytokine production. Gastroenterology 1996;111:419-25.

16. EI-Omar EM, Chow WH, Gammon MD, Vaughan TL, Risch HA, Fraumeni JF, Jr. Pro-inflammatory genotypes of IL-1 $\beta$, TNF- $\alpha$ and IL-10 increase risk of distal gastric cancer but not of cardia or oesophageal adenocarcinomas. Gastroenterology 2001; 120: (Suppl.1):A86.

17. Bayley JP, Ottenhoff TH, Verweij CL. Is there a future for TNF promoter polymorphisms? Genes Immun 2004; 5:1-15. 
18. Bonney GE, Elston RC, Correa P, Tannenbaum SR, Haenszel W, Zavala DE, et al. Genetic etiology of gastric carcinoma: II. Segregation analysis of gastric pH, nitrate, and nitrite. Genet Epidemiol 1987;4:103-14.

19. Torrado J, Correa P, Ruiz B, Zavala D, Bara J. Prospective study of Lewis antigen alterations in the gastric precancerous process. Cancer Epidemiol Biomarkers Prev 1992;1:199-205.

20. Torrado J, Ruiz B, Garay J, Cosme A, Arenas JI, Bravo JC, et al. Lewis, secretor, and ABO phenotypes, and sulfomucin expression in gastric intestinal metaplasia. Cancer Epidemiol Biomarkers Prev 1997; 6:287-9.

21. Gong C, Mera R, Bravo JC, Ruiz B, Diaz-Escamilla $\mathbf{R}$, Fontham ETH, et al. KRAS mutations predict progression of preneoplastic gastric lesions. Cancer Epidemiol Biomarkers Prev 1999;8:167-71.

22. Castaño-Molina E, Camargo M, Santacoloma M, Arango L, Villegas CR, Zuluaga D, et al. GSTT1 and not GSTM1 is associated with gastric cancer incidence in a paisa community (Colombia). Environ Mol Mutagen 2003;41(Suppl.31):167.

23. Walsh PS, Metzger DA, Higuchi R. Chelex 100 as a medium for single extraction of DNA for PCR- Based typing from forensic material. BioTechniques 1991; 10:506-13.

24. Abdel-Rahman SZ, El-Zein RA, Anwar WA, Au WW. A multiplex PCR procedure for polymorphic analysis of GSTM1 and GSTT1 genes in population studies. Cancer Lett 1996;107:229-33.

25. Di Giovine F, Camp NJ, Cox A, Chaudhary AG, Sorrel JA, Crane A, et al. Detection and population analysis of IL-1 and TNF gene polymorphisms. En: Balkwill F, editor. Cytokine molecular biology: practical approach. Third edition. Oxford: Oxford University Press; 2000.

26. González CA, Sala N, Capella G. Minireview. Genetic susceptibility and gastric cancer risk. Int J Cancer 2002; 100:249-60.

27. Kelada SN, Kardia SLR, Walker AH, Wein JA, Malkowicz SB, Rebbeck TR. The gluthation Stransferase- $\mu$ and - ? genotypes in the etiology of prostate cancer: genotype-environmental interactions with smoking. Cancer Epidemiol Biomark Prev 2000; 9:1329-34.

28. Strange RC, Lear JT, Fryer AA. Gluthation Stransferase polymorphisms: influence on susceptibility to cancer. Chem Biol Interct 1998;111-112:351-64.

29. Saadat I, Saadat M. Gluthatione S-transferase M1 and T1 null genotypes and the risk of gastric and colorectal adenocarcinoma. Carcinogenesis 2001;169:21-6.

30. Oda Y, Kobayashi M, Ooi A, Muroishi Y, Nakanishi I. Genotypes of glutathione S-transferase M1 and N- acetyltransferase 2 in Japanese patients with gastric cancer. Gastric Cancer 1999;2:158-64.

31. Kato S, Onda M, Matsukura N, Tokunaga A, Matsuda N, Yamashita K, Shields PG. Helicobacter pylori infection and genetic polymorphisms for cancer-related genes in gastric carcinogenesis. Biomed Pharmacother 1997; $51: 145-9$

32. Deakin M, Elder J, Hendrickse C, Peckman D, Baldwin D, Pantin C, et al. Gluthatione S-transferase GSTT1 genotypes and susceptibility to cancer: studies of interactions with GSTM1 in lung, oral, gastric and colorectal cancers. Carcinogenesis 1996;17:881-4.

33. Lan Q, Chow WH, Lissowska J, Hein DW, Buetow K, Engel LS, et al. Gluthatione S-transferase genotypes and stomach cancer in a population-based case-control study in Warsaw, Poland. Pharmacogenetics 2001; 11:655-6.

34. Gao CM, Takezaki T, Wu JZ, Li ZY, Liu YT, Li SP, et al. Gluthation S-transferases M1 (GSTM1) and GSTT1 genotype, smoking, consumption of alcohol and tea and risk of esophageal and stomach cancers: a casecontrol study of a high-incidence area in Jiangsu province, China. Cancer Lett 2002;188:95-102.

35. Wu MS, Chen CJ, Lin MT, Wang HP, Shun CT, Sheu JC, et al. Genetic polymorphisms of cytochrome p450 2E1, glutathione S-transferase M1 and T1, and susceptibility to gastric carcinoma in Taiwan. Int $\mathrm{J}$ Colorectal Dis 2002;17:338-43.

36. Choi SC, Yun KJ, Kim TH, Kim HJ, Park SG, Oh GJ, et al. Prognostic potential of glutathione S-transferase M1 and T1 null genotypes for gastric cancer progression. Cancer Lett 2003;195:169-75.

37. Rebbeck TR. Molecular epidemiology of the human gluthation s-transferase genotypes GSTM1 and GSTT1 in cancer susceptibility. Cancer Epidemiol Biomark Prev 1997;6:733-43.

38. Restrepo BX. Frecuencias genotípicas de los polimorfismos nulos GSTT1 y GSTM1 en las diferentes poblaciones colombianas (tesis). Bogotá: Universidad de los Andes; 2002.

39. Raunio H, Pursiainen KH, Antilla S, Hietanen E, Hirvonen A, Pelkonen O. Diagnosis of polymorphisms in carcinogen-activating and inactivating enzymes and cancer susceptibility - a review. Gene 1995;159:113-21.

40. Gaspar PA, Hutz MH, Salzano FM, Hill K, Hurtado $\mathrm{M}$, Petzl-Erler ML, et al. Polymorphisms of CYP1A1, CYP2E1, GSTM1, GSTT1, and TP53 genes in amerindians. Am J Phys Antrophol 2002;119:249-56.

41. Montecucco C, Rappuoli R. Living dangerously: how Helicobacter pylori survives in the human stomach. Nat Rev Mol Cell Biol 2001;2:457-66.

42. Sepúlveda AR. Molecular testing of Helicobacter pylori CagA status to gastric cell proliferation and apoptosis. J Clin Gastroenterol 2001;32:377-82. 
43. IARC. Infection with Helicobacter pylori. En: IARC monographs on the evaluation of carcinogenic risks to humans. Vol. 61. Schistosomes, liver flukes and Helicobacter pylori. Lyon, France: IARC; 1994. p.177-240.

44. Brenner $\mathbf{H}$, Arndt V, Stegmaier C, Ziegler $\mathbf{H}$, Rothenbacher D. Is Helicobacter pylori infection a necessary condition for noncardia gastric cancer? Am J Epidemiol 2004;159:252-8.
45. Siman JH, Forsgren A, Berglund G, Floren CH. Tobacco smoking increases the risk for gastric adenocarcinoma among Helicobacter pylori- infected individuals. Scand J Gastroenterol 2001;36:208-13.

46. Inoue M, Tajima K, Hirose K, Kuroishi T, Gao CM, Kitoh T. Life-style and subsite of gastric cancer - joint effect of smoking and drinking habits. Int J Cancer 1994; 54:494-9. 